Europhys. Lett., 15 (4), pp. 429-434 (1991)

\title{
Fragment Formation in the Break-Up of a Drop Falling in a Miscible Liquid.
}

\author{
F. T. Arecchi (*), P. K. Buah-Bassuah (**) and C. Perez-Garcia $(* * *)$ \\ Istituto Nazionale di Ottica - Largo Enrico Fermi 6, 50125 Firenze, Italy \\ (received 21 January 1991; accepted in final form 8 April 1991) \\ PACS. 47.10 - General theory. \\ PACS. 47.20 - Hydrodynamic stability and instability. \\ PACS. 68.10 - Fluid surfaces and interfaces with fluids (inc. surface tension, capillarity, \\ wetting and related phenomena.
}

\begin{abstract}
When falling in a lighter miscible solvent, a drop of liquid deforms to a torus which then breaks up into several fragments or just disappears by diffusion. By using liquids of different compositions we show the universal behaviour of the phenomenon, and its dependence on two nondimensional numbers, the fragmentation number $F$, and the Schmidt number $S$. While $F$ marks the transition from diffusion to splitting, here we show the role of $S$ in controlling the number of horizontal fragments after the first break-up. The process is explained in terms of competitions of different time scales.
\end{abstract}

The simplest example of a hydrodynamical instability in an open system is represented by the fragmentation of a drop falling in a lighter miscible liquid. Empirical reports on this phenomenon were given by several authors in the last century [1].

When the drop is falling, it is deformed into a torus. Kojima et al. [2] made some experiments and calculations on the deformation of the miscible drop and the expansion of the torus. They attributed the disagreement between calculations and experiments to a transient interfacial tension [3]. However a general understanding of the fragmentation process still lacks, because of complex intermingling of Kelvin-Helmholtz (KH) and Rayleigh-Taylor (RT) instabilities.

The torus can eventually become destabilized in some points by a RT instability, breaking up into several horizontal fragments. In the case of miscible liquids considered here, each of these fragments may undergo a similar break-up process, splitting again into secondary fragments. However, the number of successive vertical break-ups remains limited to three.

A detailed description of these phenomena including a preliminary explanation was recently presented [4]. Reference [4] is focused on the transition between no splitting and the break-up of the torus into two fragments. This is controlled by a nondimensional

(*) Also with Dipartimento di Fisica, Università di Firenze, Firenze, Italy.

(**) Permanent address: Department of Physics, University of Cape Coast, Cape Coast, Ghana.

(***) Permanent address: Departamento de Fisica, Universidad de Navarra, 31080 Pamplona and Universidad Autonoma de Barcelona, 08193 Bellaterra (Barcelona), Catalonia, Spain. 
fragmentation number defined as

$$
F=\frac{\Delta_{\ominus} V}{\mu D} g,
$$

where $g$ is the acceleration due to gravity, $\Delta \rho$ is the density difference between drop and lighter fluid (which for simplicity we call solvent), $V$ the drop volume, $D$ the mutual diffusion coefficient between the two fluids and $\mu$ is the solvent viscosity. (To simplify the problem the drop and the quiescent fluid are taken with almost the same viscosity.) The relevance of $F$ is that it is defined in terms of directly measurable quantities.

However, for a fixed liquid composition, the number of horizontal fragments increases when the drop volume increases. The aim of the present work is to characterize the formation of several (two or more) fragments after the first break-up process. To our knowledge this is the first time that this break-up process of a torus into several fragments is characterized.

Gravity and viscous drag act on a spherical drop falling in a quiescent liquid. Then the net force is the sum of a buoyancy term $F_{\mathrm{b}}$ and a drag term $F_{\mathrm{d}}$, where $F_{\mathrm{b}} \propto \Delta \rho V g$ and $F_{\mathrm{d}} \propto \mu r v, r$ being the drop radius and $v$ the drop velocity. The reference time for the drag effects is $M v / F_{\mathrm{d}}$, where $M$ is the drop mass $M=\rho V$. Skipping prefactors of the order of unity this time is given by

$$
\tau^{\prime}=\frac{\rho r^{2}}{\mu} .
$$

Therefore, the sedimentation velocity $v_{\mathrm{s}} \propto \Delta \rho g r^{2} / \mu$ is reached for times much longer than $\tau^{\prime}$.

In the experiments on glycerin/water mixtures reported in ref. [4], $\tau^{\prime}$ was much smaller than any other characteristic time, thus the drop assumed the asymptotic velocity $v_{s}$ before losing its individuality, as qualitatively sketched in fig. 1a). Here we report situations where

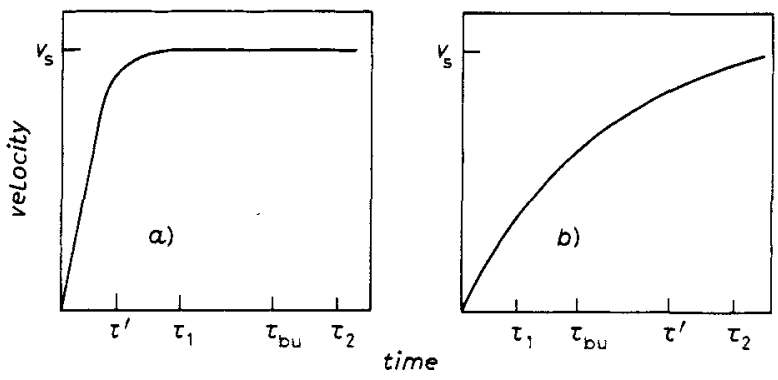

Fig. 1. - Sketch of the time evolution of the drop velocity for different time scales. a) $\left.\tau^{\prime}\left\langle\tau_{1}, b\right) \tau^{\prime}\right\rangle \tau_{1}$.

the break-up occurs before the transient duration $\tau^{\prime}$ is over, so that $v_{\mathrm{s}}$ is never reached, as sketched in fig. 1b). Besides $\tau^{\prime}$, there are two other characteristic times, namely the diffusion time $\tau_{2}=r^{2} / D$ and the time $\tau_{1}=r / v_{\mathrm{s}}$, corresponding to the transfer of $v_{\mathrm{s}}$ across the drop radius. It is easily realized that $\tau_{1}$ is the minima time for the formation of the vortex ring, hence the subsequent break-up requires a time $\tau_{b u}$ being longer than $\tau_{1}$. We have no detailed theory to evaluate the ratio $\tau_{\mathrm{bu}} / \tau_{1}$, thus we are limited to some heuristic considerations.

The parameter $F$ is defined as the ratio $\tau_{2} / \tau_{1}[4]$. Reference [4] assumed that $\tau^{\prime}$ was negligible with respect to $\tau_{2}$ and $\tau_{1}$, so that the interplay was exclusively between diffusion 
and velocity transfer to the drop. However, we should expect from Buckingham $\pi-$ theorem [5] that the break-up process is ruled by two nondimensional numbers, since the phenomenon is described by five parameters, namely, drop density $\rho$, drop-solvent density difference $\Delta p$, drop volume $V, \mu$, and $D$. We then have to build a further relevant indicator besides $F$.

The above-mentioned characteristic times suggest the following scenario. For $\tau^{\prime} \ll \tau_{1}$ the ring disappears by diffusion (fig. 2a)). If $\tau^{\prime}>\tau_{1}$ (fig. 1b)), once the ring has been formed and the circular velocity stopped, the further increasing velocity due to the still lasting transient induces a local evolution in several uncorrelated domains along the torus. This is qualitatively sketched in fig. $2 b$ ). If such a conjecture is correct, then as we decrease the ratio $\tau_{1} / \tau^{\prime}$, we should observe an increasing number of independent horizontal fragments associated with the first break-up. Thus the second nondimensional number may be defined as

$$
T=\frac{\tau_{1}}{\tau}=\frac{\mu^{2}}{g_{\rho} \Delta_{\rho} V} .
$$
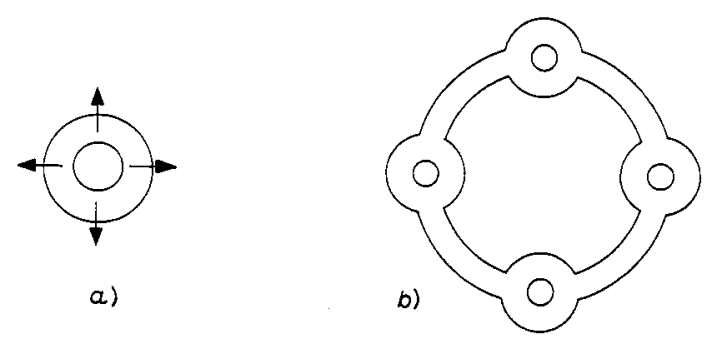

Fig. 2. - Sketch of the top view of the fluid torus evolution for different time scales. $a$ ) $\tau^{\prime}<\tau_{1}$, b) $\tau^{\prime}>\tau_{1}$.

In the laboratory practice, for a selected pair of solvent and drop compositions, we can test a variety of drop sizes. It is thus convenient to choose a different nondimensional number, which does not contain the volume. We take the remaining time ratio $\tau_{2} / \tau^{\prime}$ which happens to coincide with the Schmidt number [6]:

$$
S=\frac{\tau_{2}}{\tau^{\prime}}=\frac{\mu}{\rho D}
$$

that depends only on the fluid properties. As a consequence, the experimental results will be represented in the $F-S$ plot. Drops with a fixed liquid composition, but with different volume correspond to points along lines $F \cdot T=S=$ const, in the $F-S$ plane.

Notice that $S$ and $F$ are similar to the Prandtl and Rayleigh numbers, respectively, once the diffusion coefficient is replaced by the thermal diffusivity. Based on this analogy, we expect that an $F-S$ diagram will be a suitable parameter space to describe completely the break-up process, in analogy with the parameter space reported in ref. [7] for convective instabilities. As we decrease $S$ by controlling one of the parameters listed in eq. (4), the corresponding increase on $\tau^{\prime}$ should give rise to an increasing number of horizontal fragments.

To prove this conjecture, we have tested on several pairs of fluid compositions, each one for different drop sizes. The experimental results are shown in table I, where we have 
TABLE I.

\begin{tabular}{|c|c|c|c|c|c|c|c|c|}
\hline \multirow[t]{2}{*}{ Group } & $\begin{array}{l}\text { Percentage } \\
\text { composition }\end{array}$ & \multirow[t]{2}{*}{$\begin{array}{l}\mu \cdot 10^{2} \\
\left(\mathrm{~g} \mathrm{~cm}^{-1} \mathrm{~s}^{-1}\right)\end{array}$} & \multirow[t]{2}{*}{$\begin{array}{l}\Delta o \cdot 10^{6} \\
\left(\mathrm{~g} \mathrm{~cm}^{-3}\right)\end{array}$} & \multirow[t]{2}{*}{$\begin{array}{l}D \cdot 10^{6} \\
\left(\mathrm{~cm}^{2} \mathrm{~s}^{-1}\right)\end{array}$} & \multirow{2}{*}{$\begin{array}{l}\begin{array}{l}V \cdot 10^{3} \\
\left(\mathrm{~cm}^{3}\right)\end{array} \\
\text { IN-FIN }\end{array}$} & \multirow{2}{*}{$\begin{array}{l}\begin{array}{l}\text { Number of } \\
\text { splittings }\end{array} \\
\text { IN-FIN }\end{array}$} & \multirow{2}{*}{$\frac{F / g \cdot 10^{-3}}{\mathrm{IN}-\mathrm{FIN}}$} & \multirow[t]{2}{*}{$S \cdot 10^{-3}$} \\
\hline & $\begin{array}{l}\text { heavy fluid } \\
\text { solvent drop }\end{array}$ & & & & & & & \\
\hline
\end{tabular}

Glycerine in water

\begin{tabular}{|c|c|c|c|c|c|c|c|c|}
\hline I & 70 & 21.00 & 1.30 & 0.84 & $3-34$ & $0-2$ & $0.25-4.55$ & 209.0 \\
\hline II & 80 & 21.00 & 2.60 & 0.98 & $2-36$ & $0-2$ & $0.22-2.51$ & 193.0 \\
\hline III & 60 & 11.00 & 2.60 & 1.20 & $1-30$ & $0-3$ & $0.20-5.91$ & 77.6 \\
\hline IV & 60 & 11.00 & 3.90 & 1.25 & $1-30$ & $0-3$ & $0.28-8.51$ & 73.6 \\
\hline V & 40 & 2.20 & 3.90 & 5.30 & $1-24$ & $3-7$ & $0.33-8.03$ & 3.75 \\
\hline VI & 14 & 1.00 & 3.60 & 9.75 & $1-8$ & $6-8$ & $0.37-2.98$ & 1.00 \\
\hline \multicolumn{9}{|c|}{ Salt $(\mathrm{NaCl})$ in water } \\
\hline VII & 12 & 1.00 & 1.30 & 14.70 & $1-15$ & $3-9$ & $0.09-1.33$ & 0.63 \\
\hline \multicolumn{9}{|c|}{ Ethanol in $n$-hexane } \\
\hline VIII & $0 \quad 20$ & 0.33 & 2.58 & 9.80 & $1-14$ & $6-10$ & $0.80-11.2$ & 0.48 \\
\hline
\end{tabular}

collected the experimental data in eight groups (I to VIII). The first six groups refer to glycerin-water mixtures, the seventh (VII) goup refers to sodium chloride $(\mathrm{NaCl})$ in water and the eighth (VIII) to ethanol in $n$-hexane. For each group, we kept the fluid composition fixed as specified in the second and third columns. For instance, in the first line, 70 and 75 means that the solvent has $70 \%$ of heavier fluid which is glycerin and consequently $30 \%$ of water, whereas the drop has $75 \%$ of glycerin and consequently $25 \%$ of water. As another example, the sixth (VI) group refers to a solvent made of pure water ( $0 \%$ glycerin) with drops having $14 \%$ of glycerin and $86 \%$ of water.

Accurate calibrations of $\Delta \rho$ and $V$ have been, respectively, done with an electronic scale balance and a microsyringe. The $\mu$ values have been read or interpolated from tables [8]. The $D$ values for each pair of fluid compositions have been measured by a technique described elsewhere [9].

In order to visualize the events, we have seeded the drop with small amounts of Rhodamine $6 \mathrm{G}$ (about $10^{-9}$ mole/liter) and then observed the fluorescence by illuminating the drop with a collimated light beam. Some examples are shown in fig. 3. In this figure, we show top views of the falling drop when the break-up process begins, with the formation of a different number of fragments. (The situation when two fragments are formed can be seen

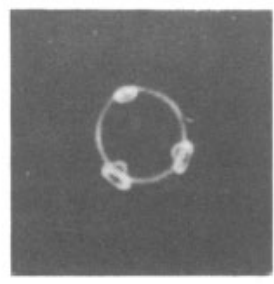

a)

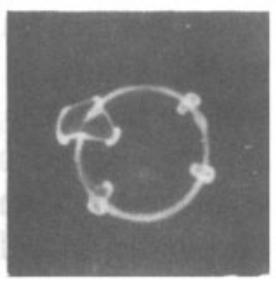

b)

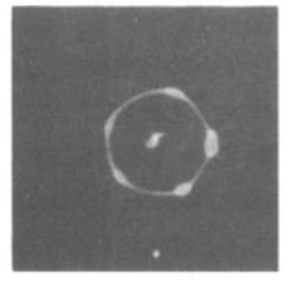

c)

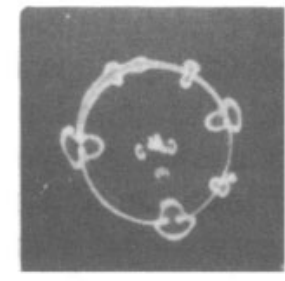

d)

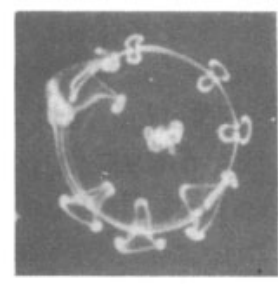

e)

Fig. 3. - Typical pictures of experimental examples showing multiple fragments after the first breakup of the torus into: $a$ ) three, $b$ ) four, $c$ ) five, $d$ ) six, and $e$ ) seven fragments. The photographs have been taken from group $\mathrm{V}$ of the experimental data classified on table $\mathrm{I}$. 


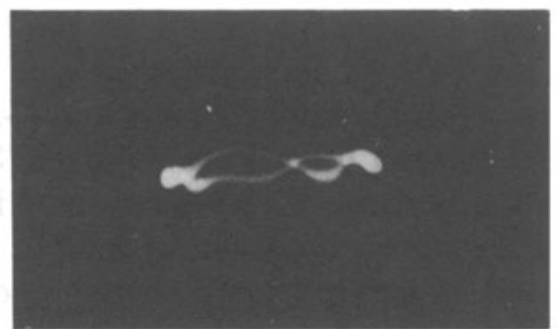

a)

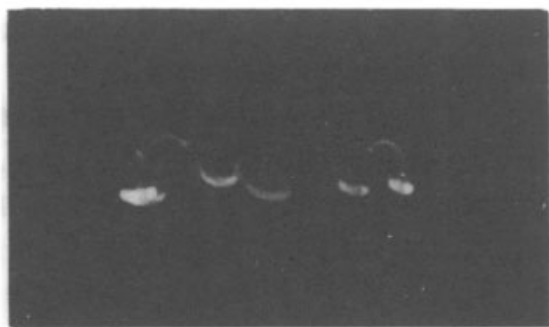

b)

Fig. 4. - Lateral views showing the successive evolution of the torus after the break-up into six fragments.

in fig. 3 in ref. [4].) In fig. 4 we give successive lateral views of a torus break-up into six fragments.

For each composition, we list the corresponding $\mu, \Delta p$ and $D$, and we test ten different volumes $V$ from an initial to a final one. In the sixth column of table I, for the sake of simplicity, we report only the two extreme values of the volume $V$ (denoted by IN-FIN). In the seventh column of table I, we report for each case the smallest and the highest number of fragments after the first break-up process within each group (for instance 6-10 in the last group). Each point on this turn is tested several times (at least ten) to check that the number

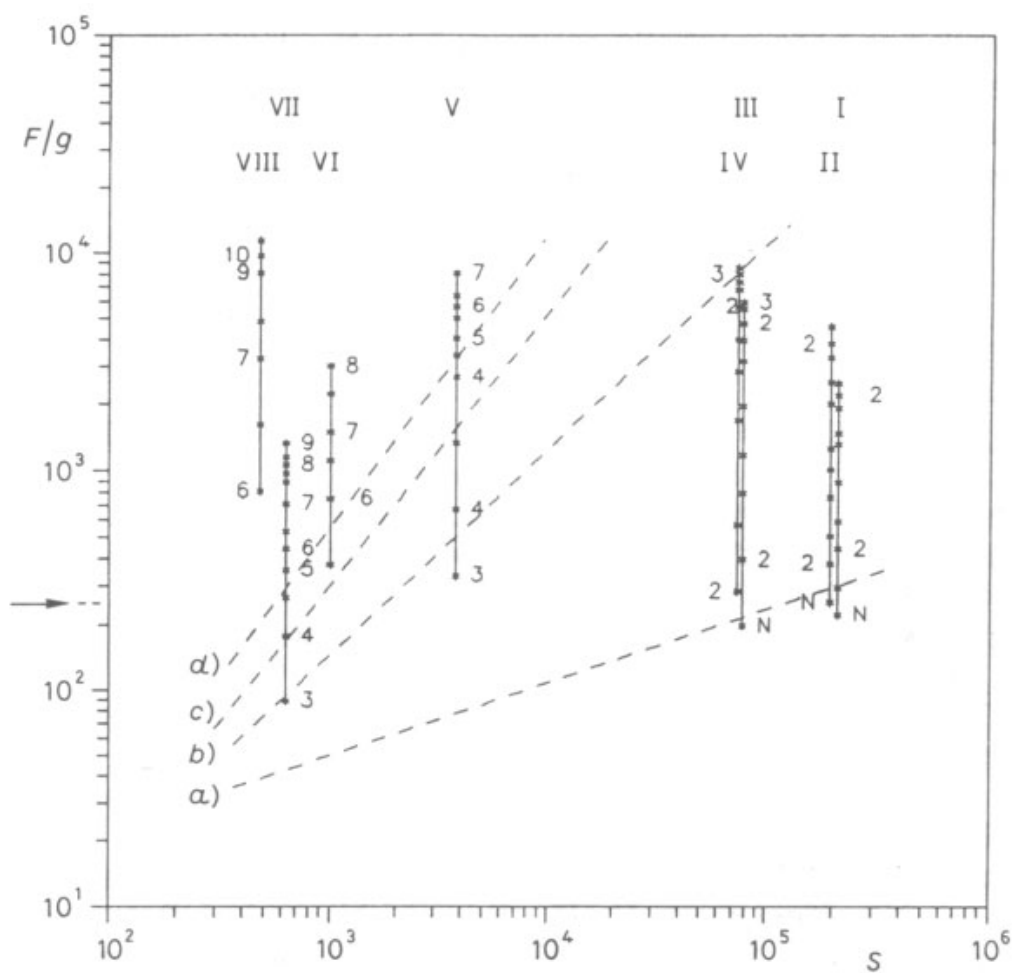

Fig. 5. $-F-S$ diagram showing the number of splittings along the vertical line of $F$-axis. The threshold lines (dashed) $a$ to $d$ ) are for $2,3,4$, and 5 fragments, respectively. I to VIII refer to different fluid compositions as specified in table $I$. The arrow denotes $F_{\mathrm{c}} / g, F_{\mathrm{c}}=(2.8 \pm 0.1) \cdot 10^{5}$ as assigned in ref. [4]. 
of fragments formed is reproducible. In the last two columns, we list $F / g$ (the lowest and highest values of each group) and $S$.

The number of fragments for the different groups of fluids are gathered in a $F-S$ diagram in fig. 5. We can draw the threshold lines, that is, dashed lines $a$ ) to $d$ ) for 2, 3,4 and 5 fragments, respectively, at the first break-up. For comparison, we have reported in fig. 5 an arrow corresponding to $F_{\mathrm{e}} / g$, with the $F_{\mathrm{c}}=(2.8 \pm 0.1) \cdot 10^{5}$ as discussed in ref. [4]. As can be seen from the $F-S$ plot, $F_{c}$ is an appropriate threshold for group I to IV corresponding to the limited range of liquid compositions previously explored [4]. As we move towards smaller $S$ values, the $S$-dependence of the first threshold (line $a$ )) becomes evident.

We have been unable to continue the $c$ ) and $d$ ) lines in the high- $F$ region, because we are limited by the calibrated drop sizes due to capillary effects in the formation of the initial drop. On the other hand, in the small-S regime, where we tested mixtures of ethyl-alcohol and $n$-hexane, the drops suffer a rapid evaporation, and the break-up process is complicated by the simultaneous presence of a large interface instability. Thus the points on column VIII are not highly reliable, even though they agree qualitatively with the other experimental groups.

In conclusion, the wide range of compositions and sizes explored, as well as the phenomenological arguments offered above, give a basis to study the formation of several fragments for the break-up process of a drop falling in a miscible fluid. These results show that the phenomenon is universal and can be characterized by two nondimensional parameters: the fragmentation number $F$ and the Schmidt number $S$.

We acknowledge fruitful discussions with Drs. F. Francini, F. Quercioli and G. PUCCIONI. This work has been partially supported by the EEC, under project SC1-0035-C and an Integrated Action (no. 49-1990) of the Italy-Spain Scientific Cooperation Programme. One of us (P.K.B-B) acknowledges the ICTP, Trieste, for financial support under Italian Laboratories Research Programme.

\section{REFERENCES}

[1] Helmholtz H., Berl. Mber, 9 (1868) 215; Philos. Mag., 36 (1868) 337; Thomson J. J. and Newall H. F., Proc. R. Soc. London, 39 (1885) 447.

[2] KoJima M., Hinch E. J. and ACRIvos A., Phys. Fluids, 27 (1984) 19.

[3] A discussion on a transient interfacial tension can be found in a recent article: JoSEPH D. D., Eur. J. Mech. B Fluids, 9 (1990) 565.

[4] Arecchi F. T., Buah-Bassuah P. K., Francini F., Perez-Garcia C. and Querciali F., Europhys. Lett., 9 (1989) 333.

[5] Duncan W. J., Thom A. S. and Young A. D., Mechanics of Fluids, 2nd edition (E. Arnold Publ. Ltd., London) 1970, p. 178.

[6] Tritton D. J., Physical Fluid Dynamics, 2nd edition (Clarendon Press, Oxford) 1988.

[7] Busse F. H., Rep. Progr. Phys., 41 (1978) 178.

[8] WEAST R. C. (Editor), Handbook of Physics and Chemistry, 52nd edition (Chemical Rubber Co., Ohio) 1971-1972, p. 215; HellweGE K. H. (Editor), Numerical Data and Functional Relationships, Landolt-Bornstein, New series, Vol. 5, Band 2 (Springer-Verlag, Berlin) 1979.

[9] ARecchi F. T., Buah-Bassuah P. K., Francini F. and QUercioli F., unpublished. 